EPJ Web of Conferences 72, 00017 (2014)

DOI: $10.1051 /$ epjconf / 20147200017

(C) Owned by the authors, published by EDP Sciences, 2014

\title{
Initial state radiation experiment at MAMI
}

M. Mihovilovič ${ }^{1, a}$, H. Merkel ${ }^{1}$, A. Weber ${ }^{1}$, P. Achenbach ${ }^{1}$, C. Ayerbe Gayoso ${ }^{1}$, T. Beranek ${ }^{1}$, J. Beričič ${ }^{4}$, J. C. Bernauer ${ }^{1}$, D. Bosnar², R. Böhm ${ }^{1}$, L. Correa ${ }^{3}$, L. Debenjak ${ }^{1}$, A. Denig ${ }^{1}$, M. O. Distler ${ }^{1}$, A. Esser ${ }^{1}$, H. Fonvieille $^{3}$, I. Friščić ${ }^{2}$, M. Gómez ${ }^{1}$, S. Kegel ${ }^{1}$, Y. Kohl', D. G. Middleton ${ }^{1}$, U. Müller ${ }^{1}$, L. Nungesser ${ }^{1}$, J. Pochodzalla1, M. Rohrbeck ${ }^{1}$, S. Sánchez Majos ${ }^{1}$, B. S. Schlimme ${ }^{1}$, M. Schoth ${ }^{1}$, F. Schulz ${ }^{1}$, C. Sfienti ${ }^{1}$, S. Širca ${ }^{5,4}$, S. Štajner ${ }^{4}$, M. Thiel ${ }^{1}$, M. Weinriefer ${ }^{1}$

(A1-Collaboration)

${ }^{1}$ Institut für Kernphysik, Johannes Gutenberg-Universität Mainz, DE-55128 Mainz, Germany

${ }^{2}$ Department of Physics, University of Zagreb, HR-10002 Zagreb, Croatia

${ }^{3}$ Clermont Université, CNRS/IN2P3, LPC, BP 10448, F-63000 Clermont-Ferrand, France

${ }^{4}$ Jožef Stefan Institute, SI-1000 Ljubljana, Slovenia

${ }^{5}$ Department of Physics, University of Ljubljana, SI-1000 Ljubljana, Slovenia

\begin{abstract}
In an attempt to contribute further insight into the discrepancy between the Lamb shift and elastic scattering determinations of the proton charge radius, a new experiment at MAMI is underway, aimed at measuring proton form-factors at very low momentum transfers by using a new technique based on initial state radiation. This paper reports on the conclusions of the pilot measurement performed in 2010, whose main goal was to check the feasibility of the proposed experiment and to recognize and overcome any obstacles before running the full experiment. The modifications to the experimental apparatus are then explained which significantly improved the quality of data collected in the full scale experiment, which was successfully executed in August 2013. At the end first findings of the new experiment are discussed.
\end{abstract}

\section{Introduction}

The proton has been scrutinized since the early days of experimental hadronic physics [1]. Its radius has been determined by various electron scattering experiments and many atomic Lamb shift measurements (see Figure 1). Both approaches gave consistent results. Unfortunately their average does not agree with the findings of recent very precise Lamb shift measurements in muonichydrogen [2, 3], which report a new value for the proton charge radius which is $7 \sigma$ away from the previously accepted value. This discrepancy, known as the proton radius puzzle, is controversial and demands further investigation. An ongoing electron scattering experiment at MAMI aims to offer new insight into this matter.

In a typical scattering experiment the radius of a proton is determined indirectly by measuring the cross-section for elastic scattering of electrons on hydrogen [5]. The measured cross-section depends on the electric and magnetic form-factors $G_{E}^{p}$ and $G_{M}^{p}$, which carry information about the charge and magnetization distribution in the proton and are extracted from the measured data via Rosenbluth separation. The charge radius is extracted from the slope of the electric form-factor at $Q^{2}=0$ :

$$
\left\langle r_{E}^{2}\right\rangle=-\left.6 \hbar^{2} \frac{d}{d Q^{2}} G_{E}\left(Q^{2}\right)\right|_{Q^{2}=0},
$$

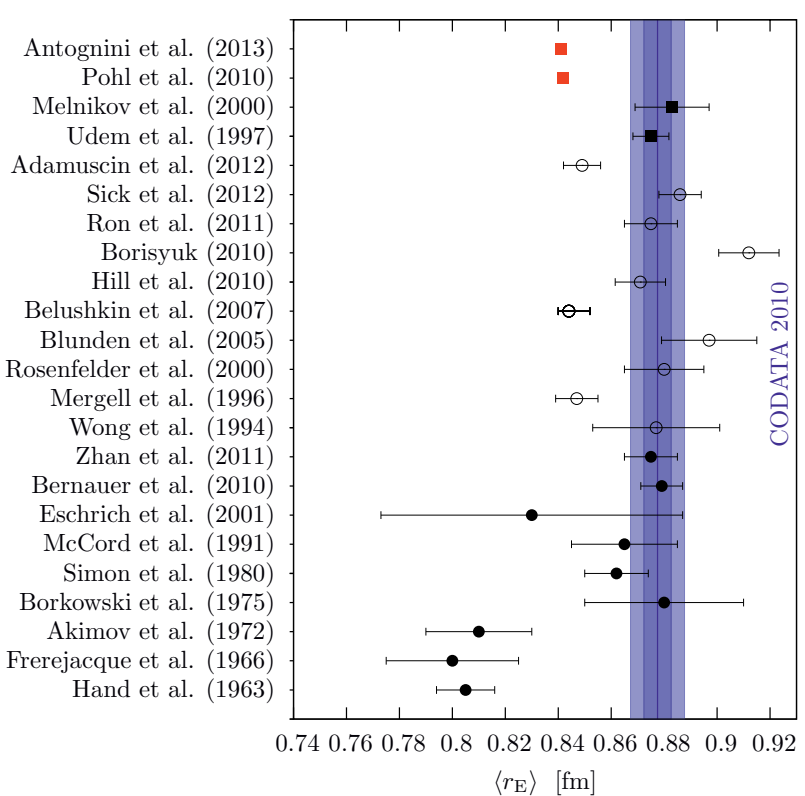

Figure 1. An overview of the existing proton charge radius results. Results of the scattering experiments are shown with full circles. Empty circles correspond to values determined by reanalysis of the existing data. Full squares represent values obtained from the Lamb shift measurements. The values determined from the muonic hydrogen measurements are colored red [4].

\footnotetext{
ae-mail: miham@kph.uni-mainz.de
} 
where $Q^{2}$ represents the square of the momentum transfer four-vector. Unfortunately, the data for $Q^{2}<$ $0.005(\mathrm{GeV} / c)^{2}$ that would allow for a reliable and precise determination of this slope do not yet exist (See Figure 2). Therefore, an extrapolation of available $G_{E}^{p}$ points to $Q^{2} \rightarrow 0$ is used to estimate $\left\langle r_{E}^{2}\right\rangle$. The extracted value of $\left\langle r_{E}^{2}\right\rangle$ is extremely sensitive to the details of this extrapolation, which in turn strongly depends on the precision and accuracy of the values of $G_{E}^{p}$ themselves.

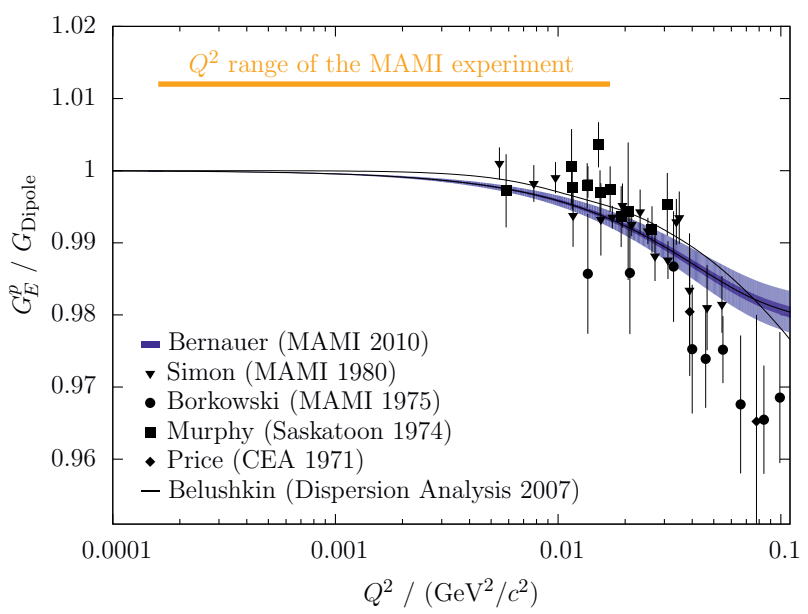

Figure 2. The proton charge form factor $G_{E}^{p}$ normalized to the standard dipole form factor $G_{D}=\left(1-\frac{Q^{2}}{0.71(\mathrm{GeV} / c)^{2}}\right)^{-1}$ as a function of $Q^{2}$. The existing data [7-11] are available only for $Q^{2}>0.005(\mathrm{GeV} / c)^{2}$. Full black line shows results of the Dispersion analysis [12]. For a reliable determination of proton charge radius, data at $Q^{2} \leq 0.005(\mathrm{GeV} / c)^{2}$ are needed. Yellow band shows the achievable $Q^{2}$ range of the new MAMI experiment.

To avoid such uncertainties, measurements of $G_{E}^{p}$ at $Q^{2}<0.005(\mathrm{GeV} / c)^{2}$ are needed. Efforts to do this are limited by the minimal accessible $Q^{2}$, which is determined by the utilized experimental apparatus. In particular, the three-spectrometer facility at MAMI [6] is on one hand bounded by the minimal scattering angle to which spectrometers can be positioned, while on the other hand it is constrained by the minimal applicable beam energy. To evade these limitations, the presented MAMI experiment tries to exploit information stored inside the radiative tail of the elastic peak in order to reach the form-factors at smaller $Q^{2}$.

\section{Initial state radiation}

The radiative tail of an elastic peak is dominated by contributions of two Bethe-Heitler diagrams [13] shown in Figure 3. The initial state radiation diagram $(\mathrm{BH}$ i) describes the process where the incident electron emits a real photon before interacting with the proton. Since the emitted photon carries away part of the incident energy, the momentum transferred to the proton $\left(Q_{\mathrm{Vertex}}^{2}\right)$ is decreased. Hence, this process opens the possibility to probe the proton structure at $Q_{\text {Det }}^{2}=Q_{\text {Vertex }}^{2}$ that are smaller than the value fixed by the experimental kinematics. On the other hand, the final state radiation diagram $(\mathrm{BH} \mathrm{f})$ corresponds to the reaction where the real photon is emitted only after the interaction with the nucleon. Consequently, $Q_{\mathrm{Vertex}}^{2}$ at the vertex remains constant, while the detected $Q_{\text {Det }}^{2} \leq Q_{\text {Vertex }}^{2}$ changes. Figure 4 shows the results of a full Monte-Carlo simulation that properly considers inelastic Feynman diagrams. For each of the kinematics the elastic peak and its two radiative tails are clearly visible. The diagonal tail corresponds to initial state radiation, while the horizontal one belongs to final state radiation. In an experi-

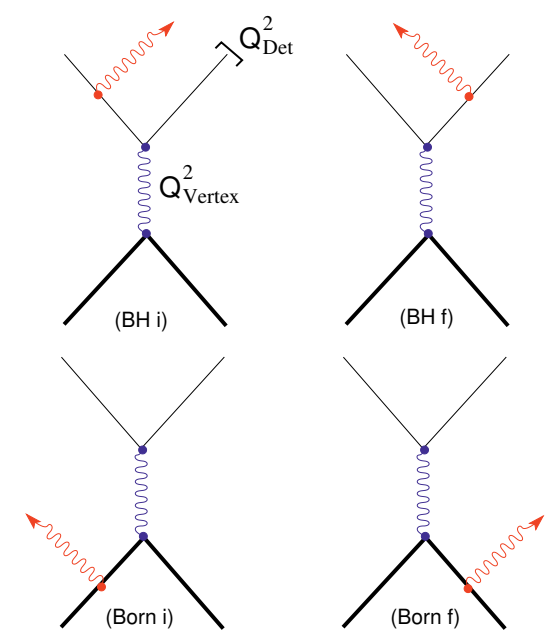

Figure 3. First order Feynman diagrams for inelastic scattering. In the Bethe-Heitler process ( $\mathrm{BH} \mathrm{i,} \mathrm{BH} \mathrm{f)} \mathrm{the} \mathrm{photon} \mathrm{is} \mathrm{emitted}$ by the electron; in Born processes (Born $\mathrm{i}$, Born $\mathrm{f}$ ) it is emitted by the proton.

ment only $Q_{\text {Det }}^{2}$ can be measured, which means that looking only at data, initial state radiation processes can not be distinguished from the final state radiation. To reach information corresponding only to the initial state radiation, measurements need to be studied together with results of a Monte-Carlo simulation. This is the basic idea of the discussed MAMI experiment, which aims to extract $G_{E}^{p}$ in the range $10^{-4} \leq Q^{2} \leq 0.005(\mathrm{GeV} / c)^{2}$.

\section{Monte-Carlo simulation}

In a detailed analysis, Feynman diagrams corresponding to Born terms (see Figure 3), where initial and final proton emit real photons, and various vertex corrections should also be considered [14-16]. These additional diagrams camouflage the form factors and make their direct extraction from cross-section measurements impossible. Therefore, an alternative approach is being employed in which data are directly compared to the simulation.

To simulate $\mathrm{H}\left(\mathrm{e}, \mathrm{e}^{\prime}\right) \gamma \mathrm{p}$ processes the Monte-Carlo simulation Simul++ is utilized, which employs a sophisticated event generator [13] that exactly calculates amplitudes for first order diagrams shown in Figure 3. Only in the next order, effective corrections to the cross-section are used. This gives Simul++ an ability to mimic real data very precisely. 


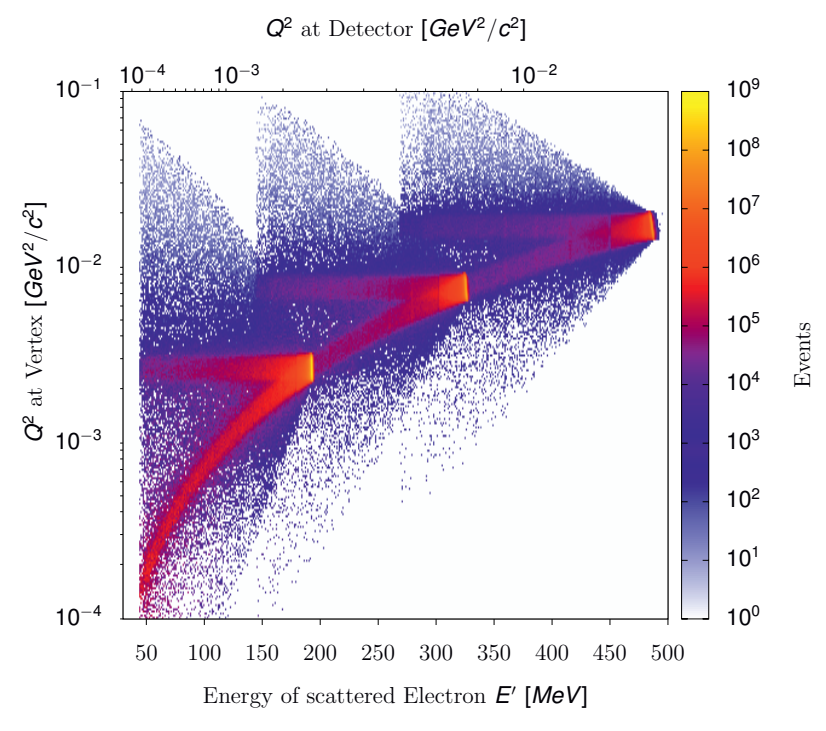

Figure 4. Results of Monte-Carlo simulation for experimental settings with beam energies $195 \mathrm{MeV}, 330 \mathrm{MeV}$ and $495 \mathrm{MeV}$. The plot shows the detected rates as a function of $Q_{\text {Vertex }}^{2}$ at the vertex and $Q_{\text {Det }}^{2}$ or energy of scattered electron which are directly measured by the spectrometer. Diagonal and horizontal tails in each settings are caused by initial state and final state radiation, respectively.

In the analysis the simulation will be run for different parameterizations of form-factors. The best value for $G_{E}^{p}$ at each $Q^{2}$ will then be determined via $\chi^{2}$-minimization of difference between the data and the simulation. The contribution of $G_{M}^{p}$ will be considered only in the estimation of the systematic uncertainty.

\section{Pilot measurement}

First measurements have been performed in 2010 using the three spectrometer setup [6] of A1 Collaboration (see Figure 5). Rastered electron beam with energies of $195 \mathrm{MeV}$, $330 \mathrm{MeV}$ and $495 \mathrm{MeV}$ was used in combination with a liquid Hydrogen target. For cross-section measurements spectrometer B was employed. It was positioned at a fixed angle of $15.25^{\circ}$, while its momentum settings were being adjusted to scan the complete radiative tail for each energy setting. Kinematic settings were chosen such that the radiative tails of all three setting overlap (see Figure 4). This enables us to test our experimental technique in the region where form-factors are well known, i.e. $G_{E}^{p}$ extracted from the radiative tail can be compared to the value obtained from the elastic peak at the coinciding $Q^{2}$. In total, 60 different setups were devised, resulting in three weeks of data taking: two weeks for production measurements and one week for empty-cell measurements, needed for proper background subtraction.

Beam currents were between $10 \mathrm{nA}$ and $1 \mu \mathrm{A}$, depending on the setup and were measured by two probes: the non-invasive Förster probe and the invasive pA-meter. Unfortunately at low beam currents and low beam energies neither of the probes is accurate enough. Hence, spec- trometer A, positioned at a fixed setting, was employed for precise monitoring of beam luminosity.

The purpose of these measurements was to test the feasibility of the proposed experiment planned for late 2013. The analysis of the data revealed that optical matrices needed for the reconstruction of the particle coordinates at the target from the directly measured detector variables were not optimal and need to be improved. The determined widths of the momentum distributions were much broader then the ideal $\delta p / p=10^{-4}$ [6].

The most critical problem exposed by the data analysis is related to the cryogenic deposition on the target cell. It consists of residual nitrogen, oxygen and water present in the scattering chamber due to insufficient vacuum conditions ( $\approx 10^{-4}$ mbar). Furthermore, it was observed that the thickness of the deposited layer changes with time (see Figure 6 (top)). The accumulated layer does not affect only particle energy losses, but changes also the detection rates and skews the luminosity measurements.

\section{Improvements to the experimental apparatus}

To overcome the problem with cryogenic depositions, a higher vacuum inside the scattering chamber had to be ensured. This was achieved by replacing gaskets and Kapton foils in the target windows. Additionally, the foils were enforced with an additional layer of Aramid foil, which allowed us to establish two order of magnitude better vacuum $\left(\approx 10^{-6}\right.$ mbar) inside the scattering chamber. This has significantly improved the measured spectra. See Figure 6 (bottom) for details. The effects of cryogens accumulating on the target walls are still visible, but effects are on the order of few percent.

The residual cryogenic depositions were monitored with spectrometer A, which was configured such that both elastic data from hydrogen needed for luminosity determination as well as elastic data from walls and cryogens were within spectrometer's acceptance. As demonstrated in Figure 7 the spectrometer has enough resolving power to clearly distinguish contributions of nitrogen and oxygen from contributions of other target elements, which allows us a precise determination of the thickness of the cryogenic layer and the corresponding particle energy-loss corrections.

To optimize optics matrices and improve the spectrometer performance for low energy running, a two week beam time was held right before the full experiment, which was dedicated to optics calibration of the spectrometers. Using these data, new optics matrices will be generated, which will then endow spectra with best possible resolutions.

To overcome the problem with the limited resolution of the existing beam current monitors, a secondary-emission monitor (SEM), was installed before the full experiment. It consists of three Havar foils, separated by $5 \mathrm{~mm}$. The outer two foils are set to a high voltage $(\approx 300 \mathrm{~V})$, while the inner foil is grounded. In the interaction with the beam the secondary electrons are emitted from the middle foil 


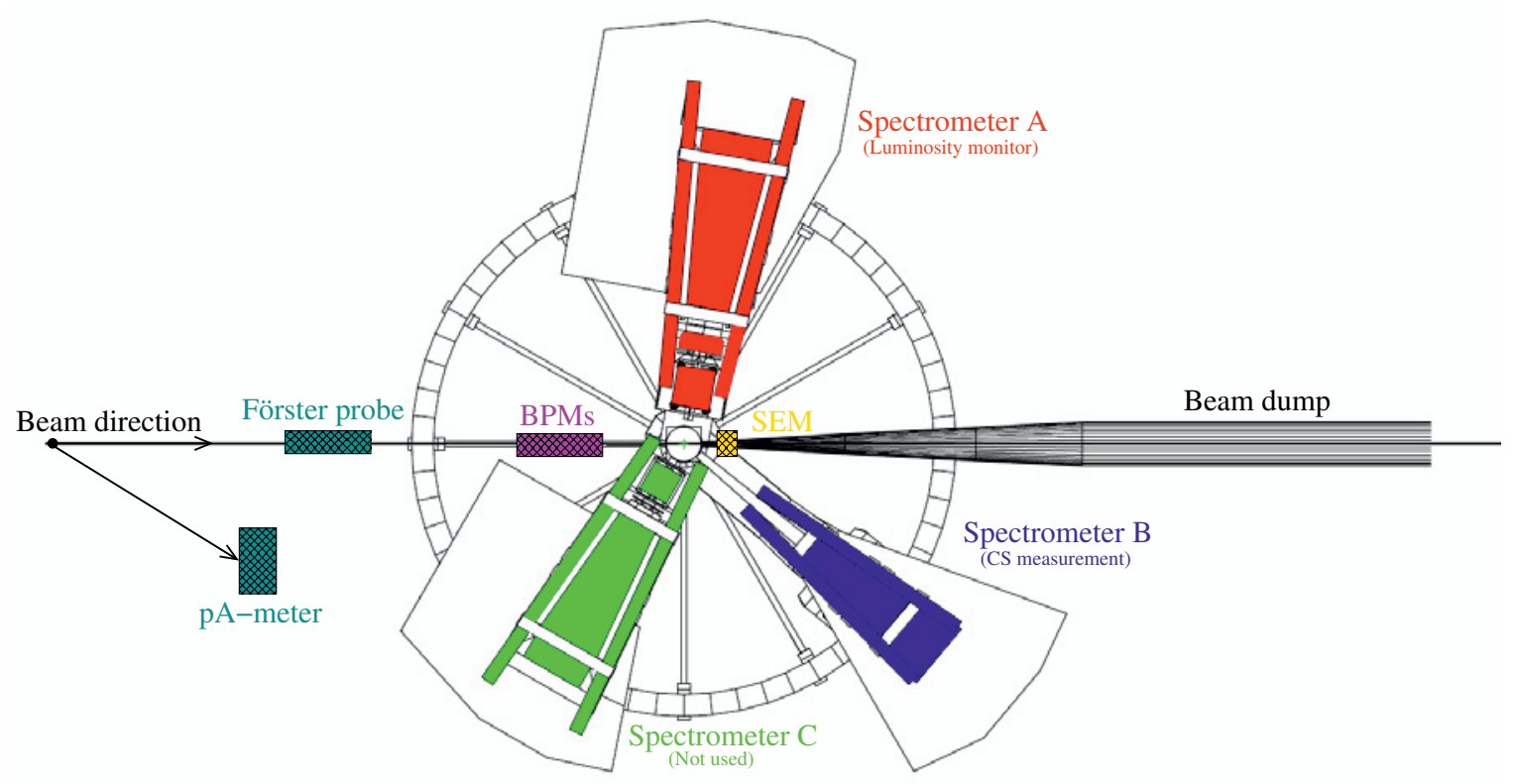

Figure 5. Three spectrometer setup at MAMI [6]. Spectrometer A is employed as luminosity monitor, while spectrometer B is used for cross-section measurements. Beam current is measured with non-invasive Förster probe and invasive pA-meter, both located inside the MAMI accelerator. Beam position is determined with two beam position monitors (BPMs) mounted right in front of the target. In the 2013 experiment a secondary electron emission monitor (SEM) was mounted in front of the beam dump as a supplementary beam current monitor.

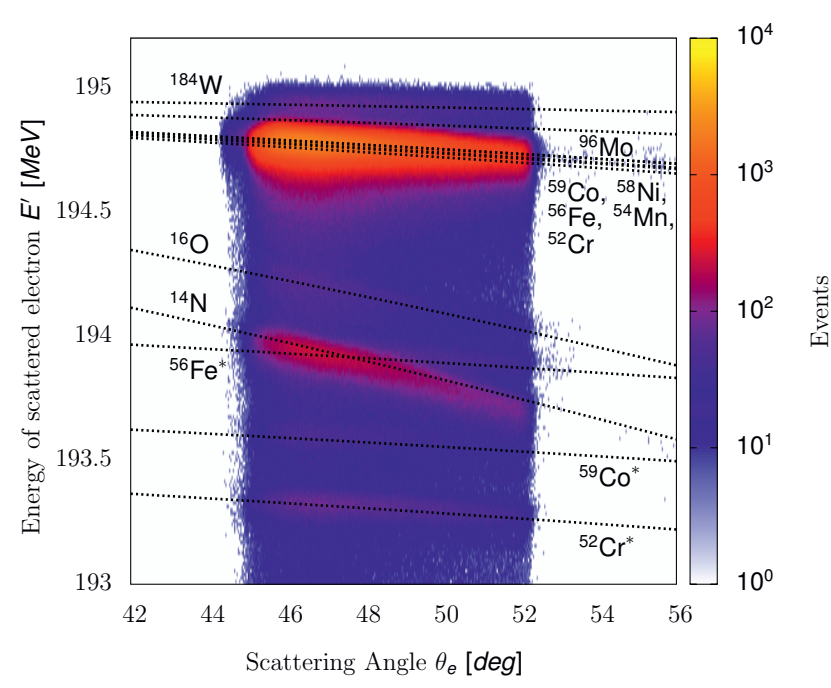

Figure 7. The distribution of scattered electrons detected by spectrometer $\mathrm{A}$ as function of scattering angle $\theta_{e}$ and energy of the detected particle $E^{\prime}$. Plot shows upper part of the measured spectrum, where energies of scattered electrons are very close to beam energy of $195 \mathrm{MeV}$. The almost horizontal bands correspond to heavy elements contained inside the Havar target walls. Electrons scattered from these elements have almost no recoil correction. The slanted bands are contributions of cryogenic nitrogen and oxygen with still observable recoil correction. The ${ }^{59} \mathrm{Co}^{*},{ }^{56} \mathrm{Fe}^{*}$ and ${ }^{52} \mathrm{Cr}^{*}$ denote first excited states of these three elements. and are drawn by the electric field towards the outer foils. The charge lost by the middle foil is proportional to the beam current and is measured by a high precision charge integrator. Such detector [17] should enable us to precisely measure very low $(\leq 10 \mathrm{nA})$ beam currents. Furthermore, being installed right next to the target (see Figure 5) it offers an opportunity to measure the actual beam current at the target, since the Förster probe and the pA-meter are both located inside the accelerator and their readings may be overestimated due to the losses of the beam transport to the experimental hall.

Unfortunately, in the experiment the SEM monitor did not perform as well as expected, even though both sensor and electronics were carefully built and installed. In spite of the extensive debugging and many calibration measurements, the desirable resolution of $\leq 1 \%$ could never be reached. Hence, we could not use it as an alternative to the standard probes but only as a supplementary device to monitor the presence of the beam in the hall.

The 2013 experiment was also the first measurement which utilized the automatic beam position control module which turned out to be a tremendous benefit to the experiment. It consists of an autonomous computer which communicates with the accelerator and every few minutes (3-5) automatically switches beam from the production continuous-wave $(\mathrm{CW})$ mode to pulse mode, makes two beam position measurements by using cavities in front of the target, and then return to the CW mode. Additionally, in between two beam position measurements it also measures the beam current by using the pA-meter. With the use of this device, an extremely reliable and stable beam of electrons was assured. 
Old data

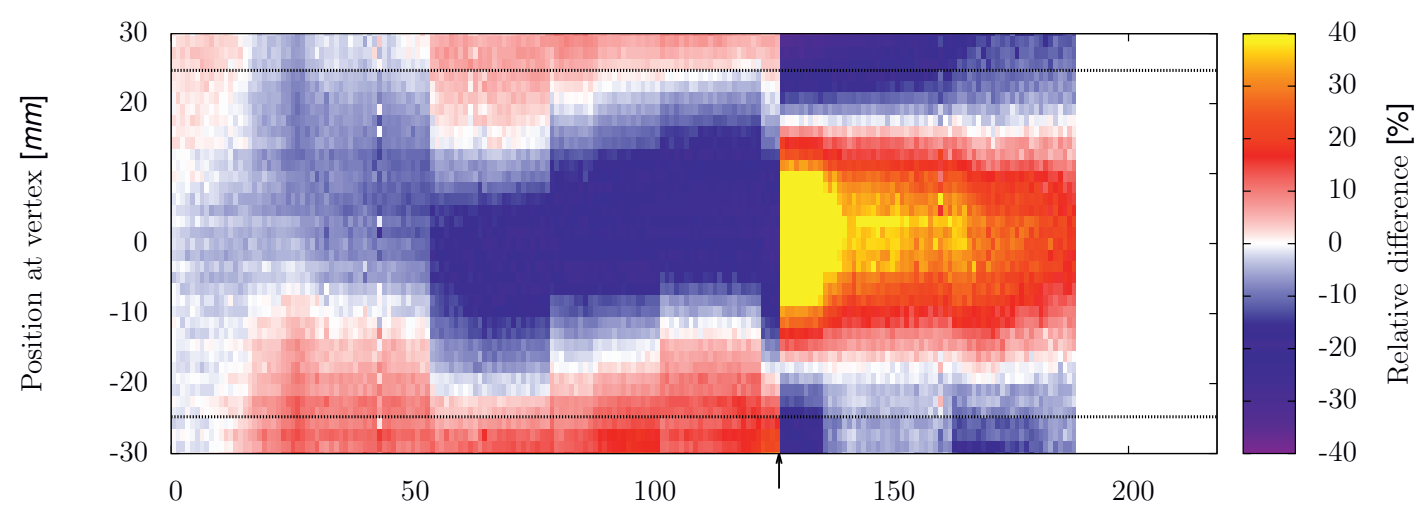

New data

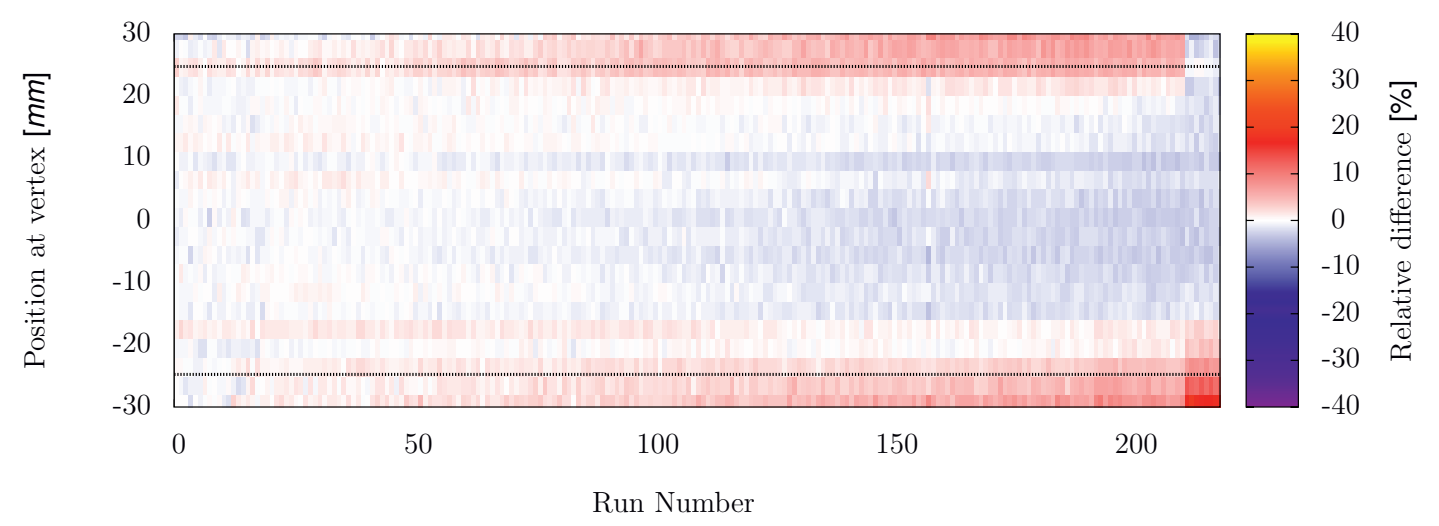

Figure 6. Top: Time evolution of the relative changes in the normalized vertex distribution during the $195 \mathrm{MeV}$ setting of the pilot measurement in 2010. As time progresses (increasing run number), more events (per run) come from target walls and less from the center of the target, which indicates that the thickness of the layer of cryogens around the target cell increases. The arrow indicates a data set taken at a higher beam current, which brought enough power to melt the cryogens. Afterwards more events are again coming from the center and less from the walls. The position of the target walls is indicated by the dashed line. Bottom: Equivalent plot obtained with the 2013 data. Effects of cryogenic depositions are still visible. However, the relative changes inside the target cell are much smaller.

\section{Full scale experiment}

The full experiment of the proton charge form-factor at MAMI was successfully performed in August 2013. The experimental setup was very similar to the one used in the 2010 pilot experiment (see Section 4), with the modification described in Section 5. Except for some minor issues related to the apparatus at the beginning of the experiment, very stable running conditions were established, which provided us with high quality data. Figure 8 shows the results of on-line analysis together with the first comparison to the simulated spectra.

The measured momentum distributions belonging to different kinematic settings are consistent and together form a continuous radiative tail. A coarse structure observed on top of the distributions is related to a changing detection efficiency and can be corrected by using calibration data. The small elastic peak on the right hand side of the main hydrogen peak corresponds to events coming from the target walls after all acceptance cuts were ap- plied. The presence of such peak indicates the need for better vertex resolution, i.e. improved optics matrices.

In order to obtain a faithful comparison of the data to the simulation, pion production processes were also considered in the Simul++ via MAID model [18]. They contribute up to $10 \%$ of the statistics in the lowest energy bins. With the inclusion of these corrections a reasonable agreement between data and simulation, with a relative difference smaller than $10 \%$ was achieved already by the online analysis. This suggests that Simul++ can satisfactorily mimic real data and that proposed approach can be successfully utilized to extract proton charged form-factor information at $Q^{2} \approx 10^{-4}(\mathrm{GeV} / c)^{2}$.

\section{Conclusions and Outlook}

The proton radius puzzle is a conspicuous open question of nuclear physics which demands further theoretical and experimental research. Therefore, a new experiment is underway at MAMI, which will provide a new prospect into 


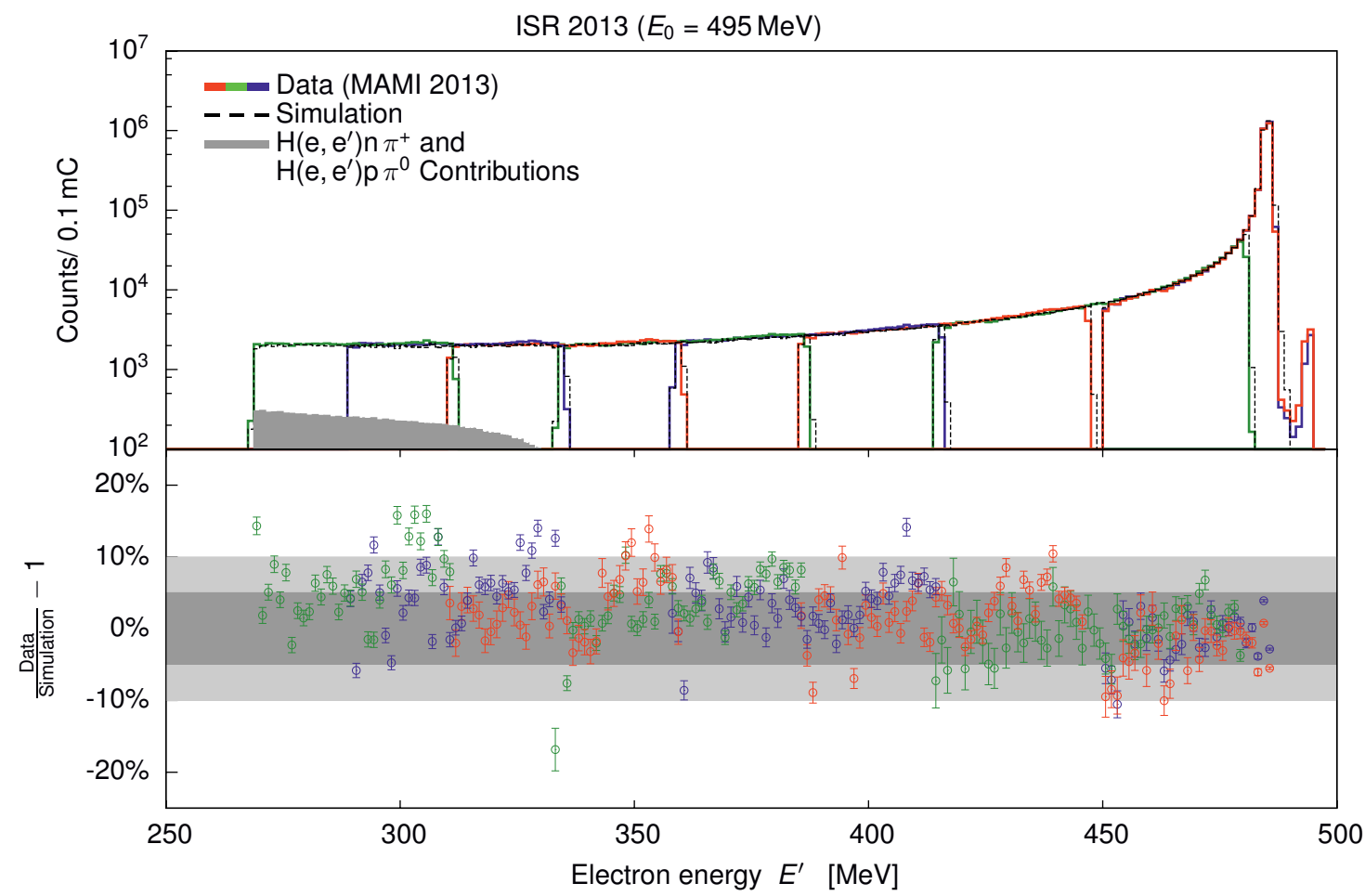

Figure 8. Top: Distributions of events as functions of energy of scattered electrons ( $\left.E^{\prime}\right)$. At the beam energy of $495 \mathrm{MeV}$ data were taken for eight different kinematic points shown on the plot with different colors. The measured spectrum was normalized to $1.0 \mathrm{mC}$ and consists of a hydrogen elastic peak and its radiative tail. A smaller elastic peak on its right corresponds to events coming from the walls and cryogens. The dashed line shows the theoretical predictions obtained by Simul++. The gray band demonstrates the contribution of the pion production processes determined with MAID [18]. Bottom: The relative differences between data and simulation for each kinematic setting. Dark and light gray bands denote $5 \%$ and $10 \%$ difference, respectively.

this matter, by measuring $G_{E}^{p}$ at very low $Q^{2}$. For that purpose a new technique is being considered, which exploits the information hidden inside the radiative tail, to determine $G_{E}^{p}$ for $Q^{2}$ as low as $10^{-4}(\mathrm{GeV} / c)^{2}$. For the experiment two sets of measurements have been made by using the three spectrometer facility of the A1-Collaboration. A pilot measurement was performed in 2010, which revealed problems mostly related to the magnetic optics of the spectrometers and to the accumulation of residual cryogenic gases around the target walls. All these handicaps were investigated and addressed before the full scale experiment, which ran in August 2013. Since then data analysis is underway and it is foreseen that the first preliminary results will be available in late 2015 .

\section{Acknowledgements}

This work is supported by the Federal State of RhinelandPalatinate and by the Deutsche Forschungsgemeinschaft with the Collaborative Research Center 1044.

\section{References}

[1] R. W. McAllister and R. Hofstadter, Phys. Rev. 102 , 851 (1956).

[2] R. Pohl et al., Nature 466, 213 (2010).
[3] A. Antognini et al., Science 339, 417 (2013).

[4] J. Beringer et al. (Particle Data Group), Phys. Rev. D 86, 010001 (2012)

[5] M. N. Rosenbluth, Phys. Rev. 79, 615 (1950).

[6] K. I. Blomqvist et al., Nucl. Instr. and Meth. A 403, 263 (1998).

[7] J. C. Bernauer et al., Phys. Rev. Lett. 105, 242001 (2010).

[8] G. G. Simon et al., Nucl. Phys. A 333, 381 (1980).

[9] F. Borkowski et al.., Nucl. Phys. B 93, 461 (1975).

[10] J. J. Murphy et al., Phys. Rev. C 9, 2125 (1974).

[11] L. E. Price et al., Phys. Rev. D 4, 45 (1971).

[12] M. A. Belushkin et al., Phys. Rev. C 75, 035202 (2007).

[13] M. Vanderhaeghen et al., Phys. Rev. C 62, 025501 (2000).

[14] L. C. Maximon J. A. Tjon, Phys. Rev. C 62, 054320 (2000).

[15] Y.-S. Tsai, Phys. Rev. 122, 1898 (1960).

[16] L. W. Tsai, Y. S. Tsai, Rev. Mod. Phys. 41, 205 (1969).

[17] S. A. Blankenburg et al., Nucl. Instr. and Meth. 39, 303 (1965)

[18] D. Drechsel et al., Eur. Phys. J. A 34, 69 (2007). 